\title{
Avaliação da capacidade de modelagem de dois sistemas reciprocantes: Reciproc Blue x V-File
}

\author{
Evaluation of the shaping ability of two reciprocating systems: Reciproc Blue $\mathbf{x} \mathbf{V}$-File \\ Evaluación de la capacidad de modelado de dos sistemas alternativos: Reciproc Blue $x$ V-File
}

Recebido: 05/11/2021 | Revisado: 11/11/2021 | Aceito: 13/11/2021 | Publicado: 24/11/2021

\author{
Maria Clara Santos Silva \\ ORCID: https://orcid.org/0000-0003-4851-437X \\ Faculdade de Ilhéus, Brasil \\ E-mail: mclaras.23@hotmail.com \\ Anna Elisa Souza Simião \\ ORCID: https://orcid.org/0000-0003-1925-293X \\ Faculdade de Ilhéus, Brasil \\ E-mail: elisaanna337@gmail.com \\ Caio Cesar Souza \\ ORCID: https://orcid.org/0000-0002-6445-5157 \\ ENDOBAHIA Cursos, Brasil \\ E-mail: caioitaobim@hotmail.com \\ Matheus Almeida Rodrigues \\ ORCID: https://orcid.org/0000-0001-9262-3704 \\ Faculdade de Ilhéus, Brasil \\ Edeilton Santana de Oliveira Júnior e Bezerra \\ ORCID: https://orcid.org/0000-0001-6766-8855 \\ Faculdade de Ilhéus, Brasil \\ E-mail: edeiltonjunior@hotmail.com \\ Adolfo de Matos de Carvalho \\ ORCID: https://orcid.org/0000-0002-4421-660X \\ Faculdade de Ilhéus, Brazil \\ E-mail: adolfodecarvalho@outlook.com \\ Ana Grasiela da Silva Limoeiro \\ ORCID: https://orcid.org/0000-0003-4633-720X \\ Faculdade de Ilhéus, Brasil \\ E-mail: grasielalimoeiro@gmail.com
}

\begin{abstract}
Resumo
Introdução: A limpeza e modelagem, são etapas de extrema importância na instrumentação dos canais radiculares para que se tenha um tratamento endodôntico feito com excelência. Com o objetivo de otimizar o tempo de trabalho e apresentar um material de qualidade, foram criadas as limas de NiTi (Niquel Titânio) que asseguram um tratamento endodôntico com sucesso. Os blocos de acrílico são usados para que tenha uma padronização dos canais simulados. Objetivo: Esta pesquisa teve como objetivo comparar as limas Reciproc Blue e V-File, após o seu segundo uso. Métodos: foram utilizados 40 blocos de acrílico, divididos em quatro grupos $(\mathrm{n}=10)$ de acordo com o número de usos de cada instrumento: REC1, REC2, VF1 e VF2. Antes do preparo do canal radicular, a patência dos canais foi verificada com uma lima $\mathrm{K}$ \#10. Imagens fotográficas do pré e pós-operatório foram obtidas para posterior sobreposição e análise em software de imagem (Adobe Photoshop CS5.1). A análise estatística foi feita utilizando teste T e o teste Mann Whitney. Resultados: mais resina foi removida do lado externo no começo da curva pelo instrumento Reciproc Blue em todos os pontos examinados ( $p<0.05)$. Em relação a distância de transporte do canal, não houve diferenças significativas em nenhum dos pontos. $\mathrm{O}$ instrumento Reciproc Blue apresentou preparos mais largos e menos conservadores. Conclusão: Ambos os instrumentos (Reciproc Blue e V-File) são seguros para o preparo de canais curvos, oferecendo uma limpeza e modelagem eficaz. Contanto o instrumento V-File apresentou preparos mais conservadores.
\end{abstract}

Palavras-chave: Canais radiculares; Níquel titânio; Preparo do canal.

\begin{abstract}
Introduction: Cleaning and shaping are extremely important steps in the instrumentation of root canals so that endodontic treatment can be carried out with excellence. With the aim of optimizing working time and presenting a high-quality material, NiTi (nickel-titanium) files have been developed to ensure successful endodontic treatment. Acrylic blocks are used to obtain a pattern of simulated canals. Objective: The aim of this study was to compare Reciproc Blue and V-File files after their second use. Methods: 40 acrylic blocks were used, divided into four groups
\end{abstract}


$(\mathrm{n}=10)$ according to the number of times each instrument was used: REC1, REC2, VF1 and VF2. Prior to root canal preparation, the patency of the root canal was checked with a \#10 K file. Pre- and postoperative photographic images were created in image processing software (Adobe Photoshop CS5.1) for later superimposition and analysis. Statistical analysis was performed using the t-test and Mann Whitney test. Results: At all points studied, the Reciproc Blue instrument removed more resin from the outside at the beginning of the curve $(\mathrm{p}<0.05)$. There were no significant differences at any of the points in terms of transport distance from the canal. The Reciproc Blue instrument had wider and less conservative preparations. Conclusion: Both instruments (Reciproc Blue and V-File) are safe for curved canal preparation and provide effective cleaning and shaping. However, the V-File instrument had more conservative preparations.

Keywords: Root canals; Nickel titanium; Canal preparation.

\section{Resumen}

Introducción: La limpieza y el modelado son pasos extremadamente importantes en la instrumentación de los conductos radiculares para que se pueda realizar un tratamiento de endodoncia con excelencia. Con el objetivo de optimizar el tiempo de trabajo y presentar un material de calidad, se crearon limas NiTi (Nickel Titanium) que aseguran un tratamiento endodóntico exitoso. Los bloques acrílicos se utilizan para tener un patrón de canales simulados. Objetivo: Esta investigación tiene como objetivo comparar archivos Reciproc Blue y V-File, después de su segundo uso. Métodos: se utilizaron 40 bloques acrílicos, divididos en cuatro grupos $(\mathrm{n}=10)$ : REC1, REC2 VF1 y VF2. Antes de la preparación del conducto radicular, se verificó la permeabilidad del conducto radicular con una lima \# $10 \mathrm{~K}$. Se obtuvieron imágenes fotográficas pre y postoperatorias para su posterior superposición y análisis en software de imágenes (Adobe Photoshop CS5.1). El análisis estadístico se realizó mediante la prueba t y la prueba de Mann Whitney. Resultados: Se eliminó más resina del exterior al comienzo de la curva mediante el instrumento Reciproc Blue en todos los puntos examinados $(\mathrm{p}<0,05)$. En cuanto a la distancia de transporte desde el canal, no hubo diferencias significativas en ninguno de los puntos. El instrumento Reciproc Blue presentó preparaciones más amplias y menos conservadoras. Conclusión: Ambos instrumentos (Reciproc Blue y V-File) son seguros para la preparación de conductos curvos, ofreciendo una limpieza y un modelado eficaces. Sin embargo, el instrumento VFile presentó preparaciones más conservadoras.

Palabras clave: Conductos radiculares; Níquel titanio; Preparación de canales.

\section{Introdução}

O objetivo do preparo do canal radicular é formatar e limpar o sistema de canais dando especial atenção à anatomia inicial, buscando um preparo cônico afunilado e manutenção do forame em sua posição original (Schilder,1974). A formatação do canal radicular é uma etapa muito importante no tratamento endodôntico, pois busca remover todo o tecido pulpar, bactérias e seus subprodutos, ao mesmo tempo em que fornece forma adequada ao canal (Schilder, 1974; Berruti et al., 2012).

A limpeza e modelagem dos canais radiculares tem como objetivo diminuir a quantidade de bactérias presentes, tornando possível uma obturação de qualidade e a cura da infecção. Sendo de extrema importância para que as próximas etapas sejam realizadas adequadamente, tornando possível o sucesso do tratamento (Perreira, 2013).

A superelasticidade da liga de Níquel Titânio (NiTi), promove a redução do risco de aberrações como formação de degrau, zip e perfurações, no entanto apresenta a desvantagem da quebra inesperada do instrumento (Giuliani et al., 2014). Atualmente os instrumentos de níquel-titânio são considerados revolucionários por apresentarem qualidades de extrema importância, como: eficiência de corte e resistência a fratura quando comparados aos instrumentos de aço inoxidável (Walia, Brantley e Gerstein, 1988; Haapasalo, 2013; Plotino et al 2014).

Em 2016 foi lançado ao mercado o sistema Reciproc Blue® (VDW, Munique, Alemanha), liga M-Wire com tratamento térmico blue, que proporciona uma maior resistência à fadiga cíclica e flexibilidade adicional, tornando possível uma progressão mais suave e segura no canal. Além disso, a lima possui como singularidade, uma secção transversal em forma de "S", apresentando duas arestas de corte e ponta ativa, na qual o instrumento se move sem empurrar, de forma que o estresse na dentina é minimizado. A Reciproc Blue® apresenta três limas (25.08, 40.06 e 50.05) com três comprimentos (21, 25 e 31mm). (Gündoğar e Özyürek, 2017).

As limas V-File são consideradas réplicas das Reciproc Blue, e também são encontradas nas numerações 25.08, 40.06, 50.05. Tem grande flexibilidade e são recomendadas para canais curvos (www.novaendovita.com) 
Em síntese, esse trabalho teve como objetivo avaliar a capacidade de modelagem dos instrumentos Reciproc Blue e V-File após o segundo uso.

\section{Metodologia}

Foram utilizados 40 blocos de acrílico (IM do Brasil, São Paulo, Brasil) que foram separados em dois grupos de limas reciprocnates: Reciproc Blue (REC-VDW, Munique, Alemanha) e V-File (TDK, China), com 20 blocos cada, e subdivididos em 4 grupos $(n=10)$ representando o primeiro e segundo uso: REC1, REC2, VF1 e VF2.

Os blocos foram envolvidos com papel laminado, para não ocorresse influência na instrumentação e depois posicionados em um torno (TORNIN, Belo Horizonte, Brasil) com a curvatura voltada para esquerda. Antes da instrumentação, os canais foram preenchidos com a tinta Nankin preta (Acrilex, China), numerados e em seguida, fotografados para registrar as formas originais dos canais.

Durante a instrumentação, todos os canais radiculares simulados foram preparados pelo mesmo operador experiente segundo a indicação dos fabricantes. Todos os canais foram preparados com motor endodôntico iRoot Pro (Bassi/ Easy Equipamentos Odontológicos, Belo Horizonte, Brasil), com os seguintes ângulos de rotação $170 \% 50^{\circ}$ graus.

Antes do preparo do canal radicular, a patência dos canais foi verificada com uma lima K \#10 (Dentsply, Maillefer). O comprimento de trabalho (CT) foi estabelecido em $17 \mathrm{~mm}$ (comprimento total do canal simulado). Cada lima foi usada com 3 movimentos de bicada e então retirada do canal e limpa com gaze. Após três movimentos, o instrumento era removido, os canais irrigados com $10 \mathrm{~mL}$ de água destilada, em seringa hipodérmica e agulha Endo-Eze (Ultradent Products, Utah, Estados Unidos) até que não houvesse detritos nos blocos. Quando a lima atingia o CT, era imediatamente removida e o canal irrigado completamente até que os detritos fossem completamente removidos.

As limas V-file foram autoclavadas antes e após o primeiro uso. Os instrumentos Reciproc Blue já vêm esterilizados de fábrica, e só foram autoclavados após o primeiro uso.

Os blocos de acrílico foram colocados na posição fixa previamente estabelecida e uma fotografia digital pósinstrumentação de cada bloco instrumentado foi tirada. As imagens pré- e pós-instrumentação correspondentes foram sobrepostas com Adobe Photoshop CS5.1 (Adobe Systems, San Jose, EUA). Os contornos dos canais foram traçados com um software experimental especialmente desenvolvido, com o qual os espaços entre os canais não preparados e preparados puderam ser medidos (Figura 1). 
Figura 1 - Sobreposição das imagens pré e pós-instrumentação marcando os pontos de medição: a - orifício do canal; b - a meio caminho entre o orifício do canal e o início da curva; c - o início da curva; d - o ápice da curva; e - ponto final do canal simulado.

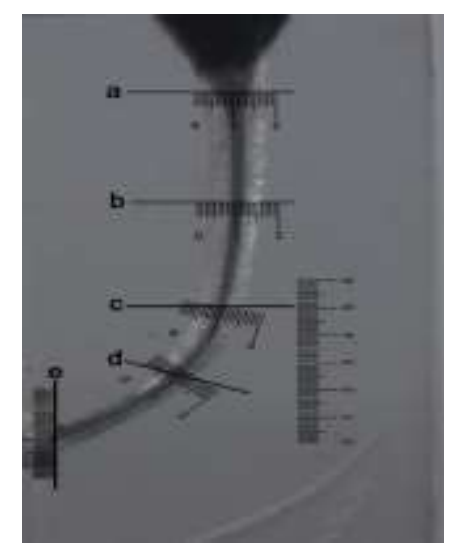

Fonte: Simião et al. 2021

Este trabalho seguiu a metodologia de Keshin, et al, 2018. Cinco pontos foram atribuídos a diferentes níveis de cada imagem do canal. O orifício do canal (a), a meio caminho entre o orifício do canal e o início da curva (b), o início da curva (c), o ápice da curva (d) e o ponto final do canal simulado (e). Os 5 pontos serviram de referência para comparar as imagens préinstrumentação e pós-instrumentação. Em cada ponto de referência, o centro do canal foi traçado medindo-se a largura do canal e dividindo-se ao meio.

Para medir a capacidade de centralização do canal, foi traçando medidas do centro do canal pré-instrumentado aos pontos do pós-instrumentados, onde foi determinado que X1 é a máxima extensão do movimento do instrumento em uma direção (lado interno da curva), o X2 é o movimento do instrumento na direção contrária e Y é o diâmetro final do preparo do canal. Em seguida foi aplicada uma fórmula Total: X1-X2/Y (Lim et al., 2013). (Figura 2).

Figura 2. X1 representa a quantidade máxima de movimento do canal para o interior da curva e X2 representa o movimento na direção oposta. Y é o diâmetro da preparação final do canal.

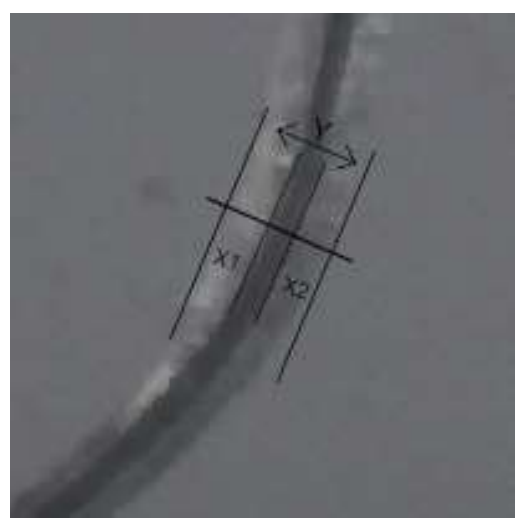

Fonte: Autoria própria.

A distância entre o centro de pré-instrumentação traçado e o ponto central pós-instrumentação foram medidos e registrados para cada um dos 5 pontos de referência; esta medição determinou a distância do transporte. Dez medições por 
ponto de referência para cada subgrupo foram calculadas em média para igualar 1 medição por nível por subgrupo e comparadas dentro de cada grupo.

Foram realizados o teste S Shapiro-Wilk, cuja finalidade foi verificar se os dados se apresentavam em uma distribuição semelhante à normal $(\mathrm{p}>0,05)$ ou não $(\mathrm{p}<0,05)$. Neste caso, verificou-se se estas variáveis estavam normalmente distribuídas.

Quando os dados se encontraram normalmente distribuídos, foi lançado mão do teste t para amostras independentes, quando não se encontravam normalmente distribuídas, foi lançado mão do teste Mann Whitney.

\section{Resultados}

Nenhum instrumento sofreu fratura durante a instrumentação dos canais simulados, mas um dos blocos que foi instrumentado pela lima V-File apresentou um desvio entre os pontos (e) e (d) (Figura 3).

Figura 3. Imagem relacionada ao desvio causado pela lima V-File.

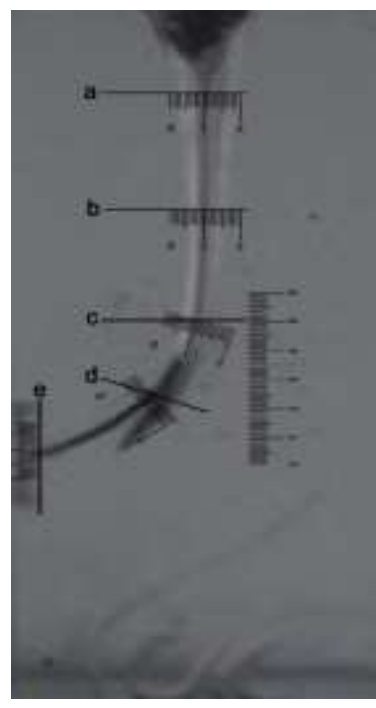

Fonte: Autores.

A Tabela 1 apresenta a largura dos 5 pontos de medição deferentes após o preparo com Reciproc Blue e V-file. O grupo V-file se mostrou mais conservador em 4 pontos, sendo eles: (a) orifício do canal; (b) meio caminho entre o orifício e o início da curva; (c) início da curva e (e) ponto final do canal. O grupo Reciproc Blue se mostrou mais conservador em 1 ponto: (d) ápice da curva.

Tabela 1. Largura total (mm) do canal em 5 pontos após o preparo do canal com 2 instrumentos.

\begin{tabular}{lccc}
\hline Ponto de medição & Reciproc Blue & V-File & p-value \\
\hline (a) Orifício do canal & $1,47 \pm 0,20^{\mathrm{a}}$ & $1,30 \pm 0,14^{\mathrm{a}}$ & 0,043 \\
\hline $\begin{array}{l}\text { (b) A meio caminho entre o orifício do canal e o } \\
\text { início da curva }\end{array}$ & $1,26 \pm 0,18^{\mathrm{a}}$ & $1,23 \pm 0,07^{\mathrm{b}}$ & 1,000 \\
\hline (c) Início da curva & $1,20 \pm 0,13^{\mathrm{a}}$ & $1,16 \pm 0,08^{\mathrm{b}}$ & 0,435 \\
\hline (d) Ápice da curva & $1,05 \pm 0,18^{\mathrm{a}}$ & $1,21 \pm 0,29^{\mathrm{b}}$ & 0,203 \\
\hline (e)Ponto final do canal & $0,76 \pm 0,18^{\mathrm{a}}$ & $0,75 \pm 0,14^{\mathrm{b}}$ & 0,889 \\
\hline
\end{tabular}

Os valores são apresentados como médias \pm desvios padrão. Letras sobrescritas diferentes indicam uma diferença estatisticamente significativa entre os grupos $(\mathrm{p}<0,05)$. Fonte: Autores.. 
A Tabela 2 apresenta a quantidade média de resina removida nos 5 pontos pelos instrumentos Reciproc Blue e V-File. No lado interno houve diferenças significantes entre as limas no ápice da curva (d). No lado externo houve uma diferença significante em todos os pontos examinados $(\mathrm{p}<0,05)$, onde a lima Reciproc Blue se mostrou menos conservadora em relação a corte.

Tabela 2. Medições da largura interna e externa $(\mathrm{mm})$ dos canais em 5 pontos de medição diferentes após o preparo do canal com 2 instrumentos.

\begin{tabular}{|c|c|c|c|c|c|c|c|c|c|c|}
\hline \multirow[t]{2}{*}{ Inst. } & \multicolumn{2}{|c|}{ (a) Orifício do canal } & \multicolumn{2}{|c|}{$\begin{array}{l}\text { (b) A meio caminho } \\
\text { entre o OC e o início da } \\
\text { curva }\end{array}$} & \multicolumn{2}{|c|}{ (c) Início da curva } & \multicolumn{2}{|c|}{ (d) Ápice da Curva } & \multicolumn{2}{|c|}{ (e) Ponto Final da Curva } \\
\hline & Interno & Externo & Interno & Externo & Interno & Externo & Interno & Externo & Interno & Externo \\
\hline $\mathrm{RB}$ & $0,45 \pm 0,15^{\mathrm{a}}$ & $0,47 \pm 0,16^{\mathrm{a}}$ & $0,43 \pm 0,15^{\mathrm{a}}$ & $0,41 \pm 0,09^{\mathrm{a}}$ & $0,43 \pm 0,09^{\mathrm{a}}$ & $0,48 \pm 0,12^{\mathrm{a}}$ & $0,42 \pm 0,12^{\mathrm{a}}$ & $0,32 \pm 0,09^{\mathrm{a}}$ & $0,29 \pm 0,14^{\mathrm{a}}$ & $0,31 \pm 0.10^{\mathrm{a}}$ \\
\hline $\mathrm{VF}$ & $0,39 \pm 0,11^{\mathrm{a}}$ & $0,35 \pm 0,14^{b}$ & $0,45 \pm 0,8^{\mathrm{a}}$ & $0,37 \pm 0,07 \mathrm{~b}$ & $0,35 \pm 0,12^{\mathrm{a}}$ & $0,47 \pm 0,09^{\mathrm{b}}$ & $0,46 \pm 0,15^{b}$ & $0,36 \pm 0,11^{\mathrm{b}}$ & $0,27 \pm 0,13^{\mathrm{a}}$ & $0,37 \pm 0,09^{b}$ \\
\hline
\end{tabular}

Os valores são apresentados como médias \pm desvios padrão. Letras sobrescritas diferentes indicam uma diferença estatisticamente significativa entre os grupos ( $\mathrm{p}<0,05)$. OC- Orifício do canal. Fonte: Autores.

A Tabela 3 apresenta a distância de transporte do canal $(\mathrm{mm})$ em 5 pontos de medição diferentes. Os grupos não apresentaram nenhuma diferença significante.

Tabela 3. Distância de transporte do canal (mm) em 5 pontos de medição diferentes após o preparo do canal com 2 instrumentos.

\begin{tabular}{lccc}
\hline Pontos de medição & Reciproc Blue & V-File & p-value \\
\hline (a) Orifício do canal & $0,00 \pm 0,10^{\mathrm{a}}$ & $0,03 \pm 0,09^{\mathrm{a}}$ & 0,430 \\
\hline (b) A meio caminho entre o OC e o início da curva & $0,01 \pm 0,15^{\mathrm{a}}$ & $0,06 \pm 0,10^{\mathrm{a}}$ & 0,361 \\
\hline (c) Inicio da curva & $-0,04 \pm 0,08^{\mathrm{a}}$ & $0,22 \pm 0,17^{\mathrm{a}}$ & 0,175 \\
\hline (d) Ápice da curva & $0,10 \pm 0,11^{\mathrm{a}}$ & $0,09 \pm 0,14^{\mathrm{a}}$ & 0,876 \\
\hline (e) Ponto final da curva & $-0,10 \pm 0,30^{\mathrm{a}}$ & $-0,14 \pm 0,17^{\mathrm{a}}$ & 0,305 \\
\hline
\end{tabular}

Os valores são apresentados como médias \pm desvios padrão. Letras sobrescritas diferentes indicam uma diferença estatisticamente significativa entre os grupos $(\mathrm{p}<0,05)$. OC - Orifício do canal. Fonte: Autores.

\section{Discussão}

O estudo teve como intuito avaliar a capacidade de modelagem de instrumentos reciprocantes usando bloco de acrílico para simulação dos canais radiculares. Em dentes naturais podem ser observadas variações significantes na anatomia, envolvendo forma, tamanho e espessura dos canais radiculares. O uso de blocos de acrílico permite uma padronização das amostras (Piazza, G. C., 2021).

A ação física dos instrumentos endodônticos sobre as paredes dos canais, e a ação química das substâncias que auxiliam na limpeza facilitando a remoção de uma grande quantidade de debris, tornando possível a recuperação e regeneração tecidual (Maranhão KM., et al, 2008).

As limas de níquel-titânio quando usadas por muitas vezes e passadas pela esterilização, acabam tendo suas lâminas deformadas o que contribui para a perda de corte. Por conta dessa fragilidade os fabricantes recomendam que o material seja descartado após o uso. Os instrumentos de NiTi TF Adaptive e Twisted File apresentaram a mesma capacidade de corte, mesmo sendo usado por 10 vezes, e não apresentaram uma diferença expressiva entre os instrumentos novos e novos e usados (Medeiros, et al 2017; Gambiarini, et al. 2016). 
Reciproc Blue apresentou maior resistência a fadiga cíclica quando comparado com o instrumento Reciproc (Keskin et al., 2017).

As limas Wave One Gold apresentaram um preparo menos conservador que as limas réplica-like W-File (Silva et al., 2021). Após o primeiro uso não houve diferenças significativas entre os pontos examinados, porém após o segundo uso os instrumentos WOG apresentaram um alargamento maior no lado interno da curva em todos os pontos, já no lado externo da curva, o grupo WF apresentou um maior alargamento em todos os pontos do canal. Após o terceiro uso não teve diferença entre os grupos concluindo que ambos removeram uma quantidade significante de resina do lado interno e externo (Carvalho., et al. 2021).

Reciproc apresentou um alargamento maior que Reciproc Blue e Wave One Gold no orifício do canal, a meio caminho entre o orifício e início da curva, no começo da curva e no vértice da curva (Keskin., et al, 2018). No presente estudo, o grupo V-File se mostrou mais conservador no orifício do canal, a meio caminho entre o orifício e o início da curva, começo da curva e forame, já o grupo Reciproc Blue se mostrou mais conservador no vértice da curva. No lado interno não houve diferenças significantes entre as limas no começo da curva (c). No lado externo houve uma diferença significante em todos os pontos, onde a lima Reciproc Blue se mostrou mais eficiente em relação a modelagem no canal anatômico. O atual estudo foi pioneiro em fazer a comparação das limas reciprocantes Reciproc Blue e V-File. Os resultados após o segundo uso mostraram que as limas Reciproc Blue são menos conservadoras, quando comparadas com a V-File.

\section{Conclusão}

Pode-se concluir que as limas Reciproc Blue e V-File são instrumentos de confiança quando se trata de canais curvos. A Reciproc Blue se mostrou mais eficiente em relação a remoção de resina, logo, menos conservadora, enquanto a V-File entregou um preparo mais conservador. Contudo, são necessários que mais estudos sejam feitos com os instrumentos para uma comparação direta.

\section{Referências}

Berutti, E., Chiandussi, G., Paolino, D. S., Scotti, N., Cantatore, G., Castellucci, A., \& Pasqualini, D. (2012). Canal shaping with WaveOne Primary reciprocating files and ProTaper system: a comparative study. Journal of endodontics, 38(4), 505-509.

Carvalho, A. de M. de; Silva, M. R. da; Rodrigues, M. A.; Oliveira Junior, E. S. de; Souza, C. C.; Rosas, C. A. P.; Cardoso, R. M.; Limoeiro, A. G. da S. (2021). Evaluation of the cutting ability of two reciprocating instruments (WaveOne Gold e W-file). Research, Society and Development, 10(14), 1-8.

Gambarini, G., Rubini, A. G., Sannino, G., Di Giorgio, F., Piasecki, L., Al-Sudani, D., \& Testarelli, L. (2016). Cutting efficiency of nickel-titanium rotary and reciprocating instruments after prolonged use. Odontology, 104(1), 77-81.

Gündoğar, M., \& Özyürek, T. (2017). Cyclic fatigue resistance of OneShape, HyFlex EDM, WaveOne Gold, and Reciproc Blue nickel-titanium instruments. Journal of endodontics, 43(7), 1192-1196.

Giuliani, V., Di Nasso, L., Pace, R., \& Pagavino, G. (2014). Shaping ability of waveone primary reciprocating files and ProTaper system used in continuous and reciprocating motion. Journal of endodontics, 40(9), 1468-1471.

Haapasalo, M., \& Shen, Y. (2013). Evolution of nickel-titanium instruments: from past to future. Endodontic topics, $29(1), 3-17$.

Keskin, C., Inan, U., Demiral, M., \& Keleş, A. (2017). Cyclic fatigue resistance of Reciproc Blue, Reciproc, and WaveOne Gold reciprocating instruments. Journal of endodontics, 43(8), 1360-1363.

Keskin, C., Demiral, M., \& Sariyılmaz, E. (2018). Comparison of the shaping ability of novel thermally treated reciprocating instruments. Restorative dentistry \& endodontics, $43(2)$

Lim, Y. J., Park, S. J., Kim, H. C., \& Min, K. S. (2013). Comparison of the centering ability of Wave. One and Reciproc nickel-titanium instruments in simulated curved canals. Restorative dentistry \& endodontics, 38(1), 21-25.

Maranhão, K. D. M., Santiago, R. D., Conde, M. S. F., \& Lamarão, S. M. S. (2008). Análise in vitro da limpeza promovida por duas técnicas de instrumentação. Salusvita, 45-54.

Medeiros, J. M. F., Haddad Filho, M. S., Habitante, S. M., da Rosa, L. C. L., Salum, G., \& Almeida, E. T. D. C. (2017). Perda de corte da lima protaper quanto a esterilização e número de uso. Revista de Odontologia da Universidade Cidade de São Paulo, 23(3), 228-237. 
Research, Society and Development, v. 10, n. 15, e177101522870, 2021

(CC BY 4.0) | ISSN 2525-3409 | DOI: http://dx.doi.org/10.33448/rsd-v10i15.22870

Pereira, H. S. C., da Silva, E. J. N. L., \& de Souza Coutinho-Filho, T. (2013). Movimento reciprocante em Endodontia: revisão de literatura. Revista Brasileira de Odontologia, 69(2), 246

Plotino, G., Rubini, A. G., Grande, N. M., Testarelli, L., \& Gambarini, G. (2014). Cutting efficiency of Reciproc and WaveOne reciprocating instruments. Journal of Endodontics, 40(8), 1228-1230.

Piazza, G. C., do Amaral Capello, P., Almeida, C. N., Blatt, J. A., \& Ferreira, R. (2021). Avaliação do número de canais modelados com limas ProDesign M até sua fratura, simulados em blocos de resina acrílica. Orbis Science, 1(1), 46-56.

Schilder, H. (1974). Cleaning and shaping the root canal. Dent Clin North Am, 18, 269-296.

Silva, M. R., Carvalho, A. M., Oliveira Junior, E.S., Souza, C. C., Rosas, C. P., Cardoso, R. M., Limoeiro, A.G.S. (2021) Estudo In Vitro da limpeza de canais simulados (Wave One Gold x W-File). Research Society and Devolopment, 10(13), 1-8.

Simeão, A. E. S., Silva, M. C. S., Oliveira Junior, E. S., Souza, C. C., Carvalho, A. M., Limoeiro, A. G. (2021) Reciproc Blue x V-File: a study on the performance of two reciprocant instruments after third use. Research Society and Devolopment 10(15), e174101522849.

Walia, H. (1988). An initial investigation of the bending and tortional properties of nitinol root canal files. $J$ Endod, 14, 246-251. 